\title{
Shared decision making and decisional conflict in the Management of Vestibular Schwannoma: a prospective cohort study
}

\author{
M. Elise Graham ${ }^{1 *} \mathbb{D}$, Brian D. Westerberg², Jane Lea ${ }^{2}$, Paul Hong ${ }^{3}$, Simon Walling ${ }^{4}$, David P. Morris ${ }^{5}$, \\ Andrea L. O. Hebb ${ }^{4}$, Rochelle Galleto ${ }^{2}$, Emily Papsin ${ }^{5}$, Maeve Mulroy ${ }^{5}$, Hannah Foggin ${ }^{2}$ and Manohar Bance ${ }^{5,6}$
}

\begin{abstract}
Background: Patients with vestibular schwannomas (VS) are faced with complex management decisions. Watchful waiting, surgical resection, and radiation are all viable options with associated risks and benefits. We sought to determine if patients with VS experience decisional conflict when deciding between surgery or non-surgical management, and factors influencing the degree of decisional conflict.
\end{abstract}

Methods: A prospective cohort study in two tertiary ambulatory skull-base clinics was performed. Patients with newly diagnosed or newly growing vestibular schwannomas were recruited. Patients were given a demographic form and the decisional conflict scale (DCS), a validated measure to assess the degree of uncertainty when making medical decisions. The degree of shared decision making (SDM) experienced by the patient and physician were assessed via the SDM-Q-10 and SDM-Q-Doc questionnaires, respectively. Non-parametric statistics were used. Questionnaires and demographic information were correlated with DCS using Spearman correlation coefficient and Mann-Whitney U. Logistic regression was performed to determine factors independently associated with DCS scores.

Results: Seventy-seven patients participated (55\% female, aged 37-81 years); VS ranged in size from 2 mm-50 mm. Significant decisional conflict (DCS score 25 or greater) was experienced by 17 (22\%) patients. Patients reported an average SDM-Q-10 score of 86, indicating highly perceived level of SDM. Physician and patient SDM scores were weakly correlated ( $p=0.045$, Spearman correlation coefficient 0.234). DCS scores were significantly negatively correlated with a decision to pursue surgery, presence of a trainee, and higher SDM-Q-10 score. DCS was higher with female gender. Using logistic regression, the SDM-Q-10 score was the only variable associated with significantly reduced DCS.

Conclusions: About one fifth of patients deciding how to manage their vestibular schwannoma experienced a significant degree of decisional conflict. Involving the patients in the process through shared decision-making significantly reduced the degree of uncertainty patients experienced.

Keywords: Decisional conflict, Acoustic neuroma, Shared decision making, Vestibular schwannoma

\section{Background}

Vestibular schwannomas (VS) have an estimated incidence of $0.6-1.9$ per 100,000 annually [1]. In tertiary neurotology practices, however, they can be a substantial part of the patient flow. Symptoms are variable, and it is therefore difficult to create a standardized management algorithm. Options include watchful waiting, stereotactic radiation

\footnotetext{
* Correspondence: Elisegraham.md@gmail.com

${ }^{1}$ Division of Otolaryngology - Head and Neck Surgery, Western University and London Health Sciences Centre, 5010, 800 Commissioners Road E, London, Ontario, Canada

Full list of author information is available at the end of the article
}

surgery (SRS) and conventional surgical approaches. The risks and benefits of each option may make management decisions challenging for both surgeons and patients.

Watchful waiting is a commonly employed treatment approach in the modern management of vestibular schwannomas. There are no identified factors to predict an individual's VS growth pattern at initial presentation or when the tumor first shows evidence of growth [2,3]. Various series have shown that the majority $(>60 \%)$ of VSs do not grow if followed. If the tumor does grow, most grow slowly though some grow rapidly, with an overall estimated

(c) The Author(s). 2018 Open Access This article is distributed under the terms of the Creative Commons Attribution 4.0 International License (http://creativecommons.org/licenses/by/4.0/), which permits unrestricted use, distribution, and 
annual growth between 0.4 and $2.9 \mathrm{~mm}$ per year [4-6]. When growth does occur, it can affect patient function and influence both the options and outcomes for future management.

Microsurgical resection is another management option for patients, via the translabyrinthine, retrosigmoid or middle cranial fossa approach, with each possessing its own set of advantages and disadvantages. Surgery places surrounding nerves and arteries at potential risk. Standard neurosurgical risks also apply, such as cerebrospinal fluid leak and meningitis [7]. Stereotactic radiation surgery (SRS) as an option has its own risks. SRS can cause acute hydrocephalus from tumor swelling, albeit rarely [8], or contribute to hearing loss $[9,10]$. If SRS fails, salvage surgery may be more challenging [11]. There is also the low but real risk of malignant transformation in the radiation field $[12,13]$.

With multiple treatment options, all with their own inherent risks and benefits, and variable natural history, any treatment decision is complex and likely to involve significant anxiety and stress. The decision between treatment options must consider the patient's experiences, values and risk tolerance, in addition to tumor characteristics and local physician expertise. Shared decision-making (SDM) may play an important role in facilitating the management of VS. SDM is a collaborative approach that describes the process of patients working with healthcare providers to come to a consensus regarding their care. SDM has previously been shown to decrease uncertainty around management decisions and improve health-related quality of life $[14,15]$.

A related topic is "decisional conflict", which defines difficulties experienced by patients in coming to a decision regarding their care. Previous data has suggested that the degree of decisional conflict experienced by patients may be influenced by the degree of shared decision-making in patient consultation [16-18], with those patients perceiving more SDM experiencing less decisional conflict. Studies in other surgical decision-making contexts have showed that patients experience significant decisional conflict when deciding between surgical and non-surgical treatment for various conditions $[17,19,20]$.

The objective of this study was to determine if patients experience decisional conflict when making management decisions for their VS, and to explore which factors, if any, influence the degree of conflict experienced. Given the benign nature of the tumors and the sometimes conflicting and confusing evidence surrounding management of patients with VS, we hypothesized that these patients may experience significant levels of decisional conflict.

\section{Methods}

\section{Ethical considerations}

Institutional ethics board approval was obtained at both participating centers. Written informed consent was obtained from each patient and de-identified data was securely stored.

\section{Aim}

To assess the degree of shared decision making experienced by patients when deciding how best to manage their VS.

\section{Participants}

This study was carried out in two tertiary/quaternary academic centres. One centre (Halifax, Nova Scotia) involved a multi-disciplinary skull base clinic with fellowship trained neurotologists and neurosurgeons, whereas the second site (Vancouver, British Columbia) involved a neurotology clinic with fellowship trained neurotologists with referrals to other services being directed after the initial consultation.

All new patients with a clinical diagnosis of VS presenting to the two centers were approached. Follow-up patients with demonstrated recent VS growth on serial MRI scans were also approached, as they were faced with the need to make a new treatment decision. Patients were excluded if they declined to participate or were not fluent in English. Patients were not approached if they had previously undergone treatment of their VS.

All patients underwent a standardized clinic visit, which involved a neurotological history and examination, review of imaging, discussion of the diagnosis, and a review of possible treatment options, highlighting those felt to be most appropriate for the individual patient. Risks and benefits of each option were discussed by the attending surgeons. Following the consultation, patients who agreed to participate were referred to the research assistant who provided details of the study, obtained consent and administered the questionnaires to the patients.

\section{Measures \\ Demographic form}

Baseline demographic information was collected, including previous surgeries, education, and income. A separate demographic form was completed by the attending surgeons responsible for the consultation including maximum diameter of VS measured on MRI, presenting symptoms, presence of a trainee, and management options presented.

\section{Decisional Conflict Scale (DCS)}

This 16-item Likert-like measure is a validated scale that determines patient uncertainty about a medical decision. Sample items include the following: "I am clear about what benefits matter most to me," "I feel sure about what to choose," and "My decision shows what is important to me." It includes five subcategories, is context non-specific, and has been used in a variety of surgical settings [17-20]. Previous research has suggested that a score of 25 or greater is representative for significant decisional conflict [12]. 


\section{Shared Decision Making Questionnaire-Patient Version (SDM-Q-9)}

This is a validated measure with nine items on a Likert scale to assess the perception of patients regarding their involvement in clinical decision-making. Total score range from 0 (no shared decision-making) to 100 (a high degree of shared decision-making). Sample items include: "My doctor and I selected a treatment option together" and "My doctor made it clear that a decision had to be made." The SDM-Q-9 has been shown to have high reliability [19].

\section{Shared Decision Making Questionnaire-Physician Version (SDM-Q-Doc)}

This is a validated scale that was developed from SDM-Q-9 to make it applicable for healthcare providers. Its format is similar to the SDM-Q-9 with overall score also ranging from 0 to 100. This scale has demonstrated high reliability [20]. Sample items include: "I wanted to know exactly from my patient how he/she wants to be involved in making the decision" and "I told my patient that there are different options for treating his/her medical condition."

\section{Data analysis}

Power calculation indicated that 52 patients would be required to detect a correlation coefficient of 0.38 between shared decision-making and decisional conflict. This correlation coefficient has been established in previous research examining DCS and SDM [15]. To ensure adequate sample size, accounting for attrition and incomplete data, we set the recruitment goal at 75 patients.

Data entry was completed in Microsoft Excel ${ }^{\mathrm{m}}$ and analysis conducted in RStudio, Version 1.0.136 (Boston, MA).

DCS was not normally distributed; therefore, non-parametric statistics were used. Descriptive statistics such as median, interquartile range and standard error (SE) are reported. Scores greater than 25 on the DCS indicate significant decisional conflict. Mann-Whitney $U$ test and Spearman's correlation coefficient were utilized to correlate DCS with demographic variables. Spearman's correlation coefficient was used to compare SDM-Q-9 and SDM-Q-Doc. Logistic regression was performed to determine which factors are independently associated with decisional conflict. Statistical significance was set at $p<0.05$.

\section{Results}

\section{Patient demographics}

Seventy-seven patients participated, 62 of whom (79\%) were presenting to the clinics for the first time, while 15 (19\%) were follow-up patients with demonstrated VS growth. One patient did not have new/follow-up patient status recorded. Fifty-eight percent of patients were recruited from Halifax, with the remainder were from Vancouver. Seventy-one percent of patients $(55 / 78)$ had undergone surgery previously, with the most common surgery being a hysterectomy. Average patient age was 57.8 years old (range 37 to 81 years); $55 \%$ of patients were female and the majority (73\%) were married. Patients had an average of 14 years of education (range 8 to 20 years).

\section{Vestibular schwannoma characteristics}

The participants' mean maximal diameter of vestibular schwannoma was $18.3 \mathrm{~mm}$, with diameters ranging from $2 \mathrm{~mm}$ to $50 \mathrm{~mm}$. The most common presenting complaint was hearing loss, noted in $92 \%$ of patients (72/78). Other presentations included vestibular dysfunction in $44 \%$, tinnitus in $19 \%$, and facial numbness in $17 \%$.

\section{Decisional conflict}

Median decisional conflict across all patients was 4.69. Seventeen participants (22\%) had significant decisional conflict, as defined by a score of 25 or more (Fig. 1). Thirty-two patients $(41 \%)$ reported that they experienced zero decisional conflict. The DCS score did not significantly differ based on previous surgery, marital status, study site, or type of visit (first time vs. follow-up). Significant differences were noted in DCS scores between participants who decided upon surgery as a treatment and those who did not $(p=0.034)$, with the surgery group experiencing lower decisional conflict. There was also a significant difference noted between the degree of decisional conflict reported by female patients and male patients (male lower), and lower DCS scores were noted in those encounters where a trainee was present $(p=0.035)$.

DCS scores were not related to whether the patient felt they wanted surgery prior to the consultation, patient age, number of previous surgeries, years of education, size of VS, or number of symptoms. DCS was also not correlated with any individual presenting symptom, including hearing loss, vestibular dysfunction, tinnitus and facial numbness $(p>0.05)$.

\section{Shared decision-making}

Median SDM-Q-9 scores for patients was 88.89. Median SDMQ score for physicians was 75.56 (Fig. 2). There was a significant difference between patient and physician SDMQ scores $(p<0.001)$. The mean difference in score between physician and patients was 11.29 (95\% CI, 7.98-14.61). Spearman's correlation between participant and physician shared decision-making scores was 0.234 , $p<0.05$, indicating they were weakly correlated (Fig. 3).

A significantly negative correlation between SDM-Q-9 scores and DCS scores was noted (Fig. 4). SDM-Q-Doc scores and DCS scores were not correlated.

\section{Management decisions and decisional conflict}

More than half of patients $(40 / 78,51 \%)$ decided on a watchful waiting approach. Twenty-one patients (27\%) decided to 


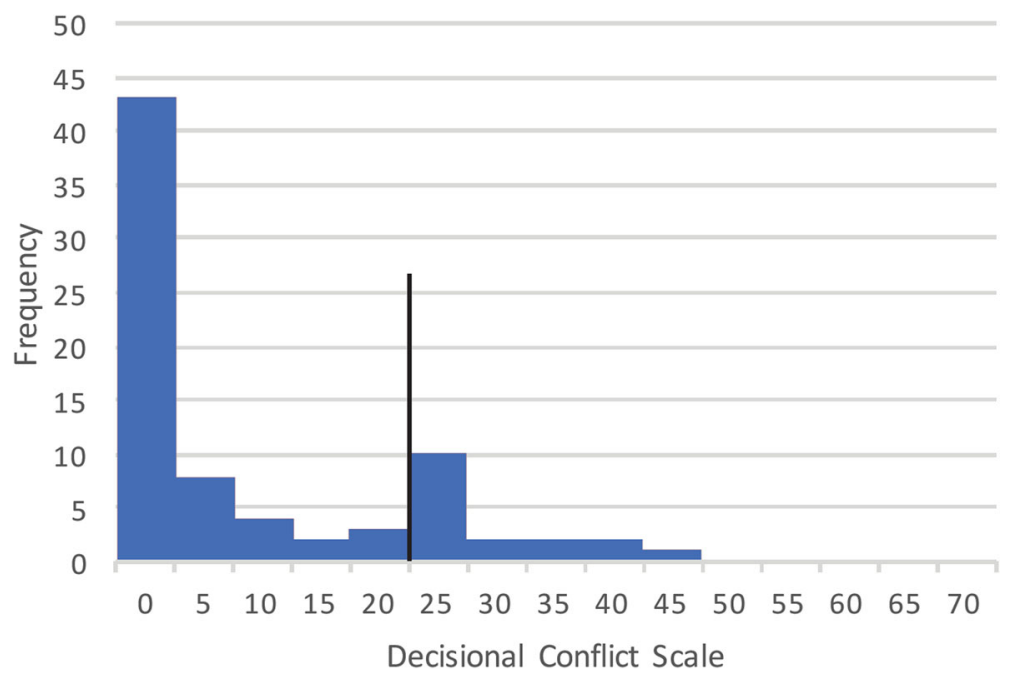

Fig. 1 Distribution of decisional conflict amongst patients. The black line represents significant decisional conflict (25), and 22\% of patients exceeded this

proceed with surgery, and 8 patients $(10 \%)$ decided on radiation. More than one option was usually discussed with each patient. Options discussed with patients were radiation with $88.3 \%$ of patients, surgery with $93.5 \%$, and watchful waiting (W \& W) with $85.7 \%$. Seventy-one of 75 patients (95\%) who responded to this question indicated that they knew surgery was an option prior to the consultation. Figure 5 shows the distribution of DCS by treatment decision.

\section{Logistic regression}

Logistic regression was performed to predict the presence of significant decisional conflict ( $\mathrm{DCS}>25$ ). The best model

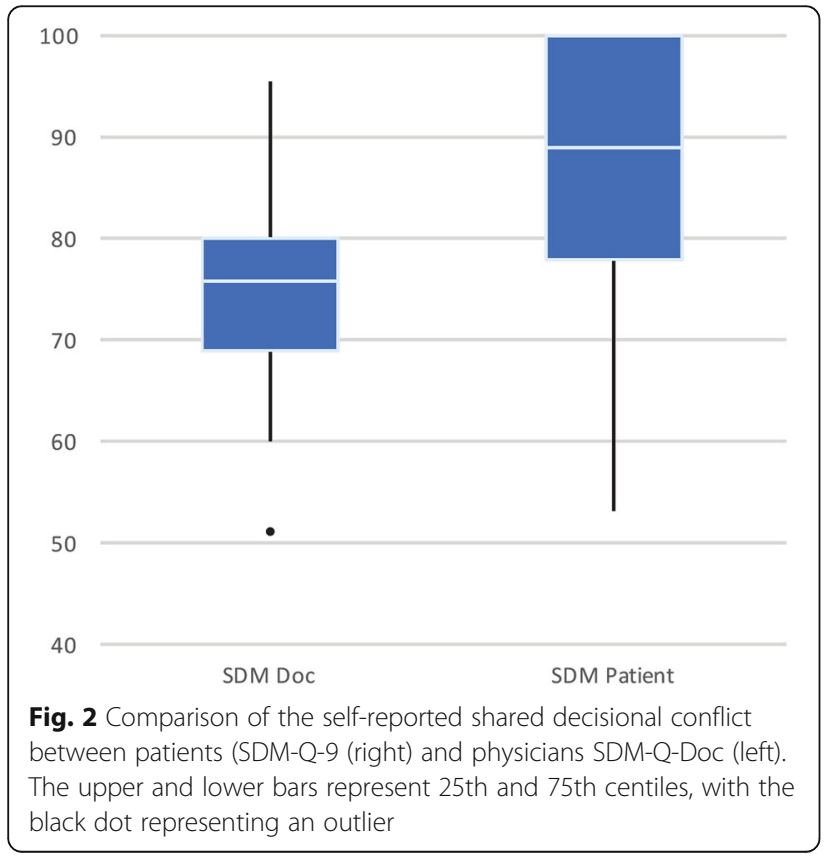

generated, by lowest Akaike information criterion (51.77), incorporated patient sex, age, number of previous surgeries, years of education, SDM-Q-10 score and number of options discussed with the patients to predict the presence or absence of significant decisional conflict. Of the individual variables, only the patient's SDM-Q-10 score contributed significantly to prediction, with higher SDM-Q-10 scores predicting lower probability of significant decisional conflict.

\section{Discussion}

\section{Synopsis of key findings}

Given the benign nature of VS and the sometimes conflicting and confusing evidence surrounding management of VS, we hypothesized that these patients may experience significant levels of decisional conflict. The overall median decisional conflict in our study at 4.69 was well below the cut-off for significant decisional conflict. However, one of every five patients (22\%) experienced significant decisional conflict $(\mathrm{DCS}>25)$. Previous studies suggest that decisional conflict affects emotional wellbeing and may influence subsequent regret surrounding their management choices [21, 22].

In deciding whether to proceed with an intervention or conservative management for their VS, patients must weigh significant risks to hearing, balance, and facial nerve function. Risks of watchful waiting are that that growth may limit the ability to use SRS or that the patient may experience a general decline in health that increases the risks of surgery later. Because facial nerve and hearing outcomes from surgery are related to size of the tumor there are risks that the outcome will not be as optimal if the tumor grows prior to surgery [23]. Rates of growth are variable and not predictable. Not surprisingly, because we can only provide patients with probabilistic outcomes rather than 


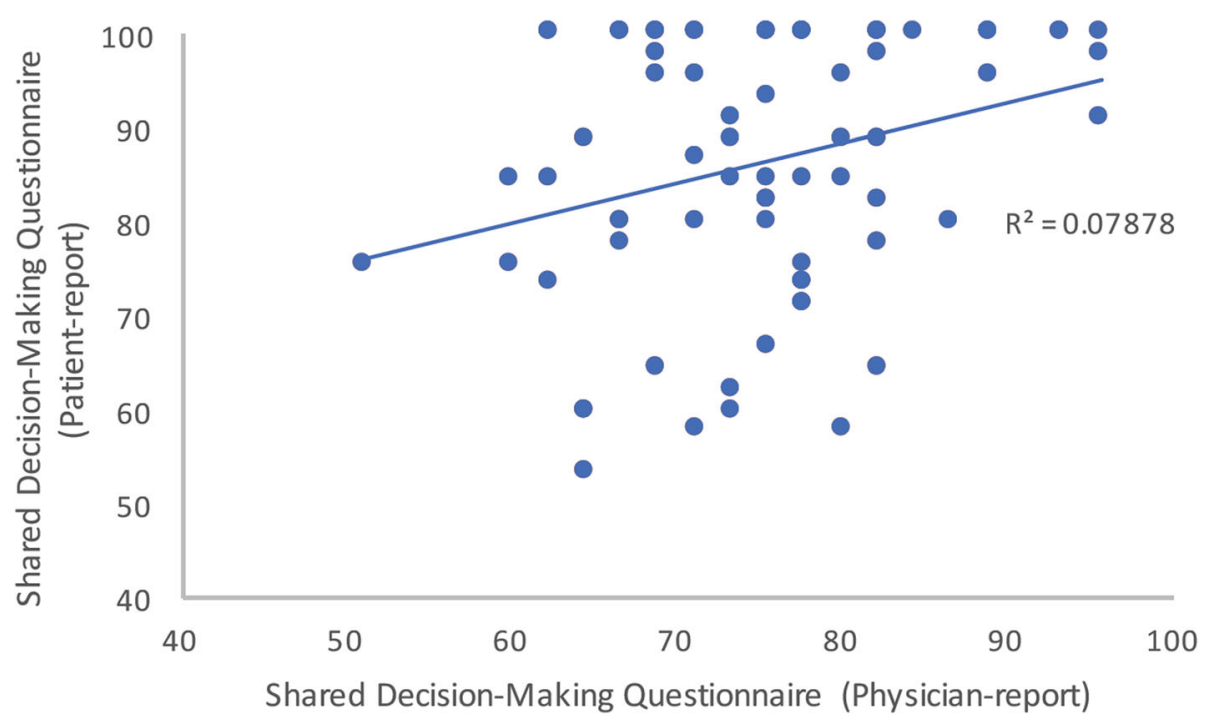

Fig. 3 Scatter plot of the correlation between patients' and physicians' perceptions of shared decision making (SDM-Q-9 and SDM-Q-Doc). The $R^{2}$ value is shown, correlation coefficient is 0.234

individualized precise trajectories, we often encounter significant anxiety associated with the watchful waiting choice. This has not been well explored in previous literature.

This study suggests, as in previous studies, that patient and physician estimates of SDM were not well correlated (correlation coefficient 0.234 ) $[15,18,24]$. Patients overall rated a higher level of SDM than physicians did. Physicians do not seem to have an accurate sense of how involved their patients felt, although in this case the physicians underestimated their success in sharing the clinical decision. After logistic regression, shared decision making was the only factor that was significantly correlated with reduced decisional conflict. This concept clearly must become a focus of clinical consultation to improve patient experience. Physicians should examine critically their technique for presenting management options, when equivocal, to involve the patients in deciding how to manage their care.

A novel finding in our study is that the presence of trainees in the consultation appeared to decrease the degree of decisional conflict experienced by patients. The presence of a trainee may remind the consultant to use non-medicalized language, or result in additional repetition of information if the trainee discusses management options prior to the consultant entering the room. Further

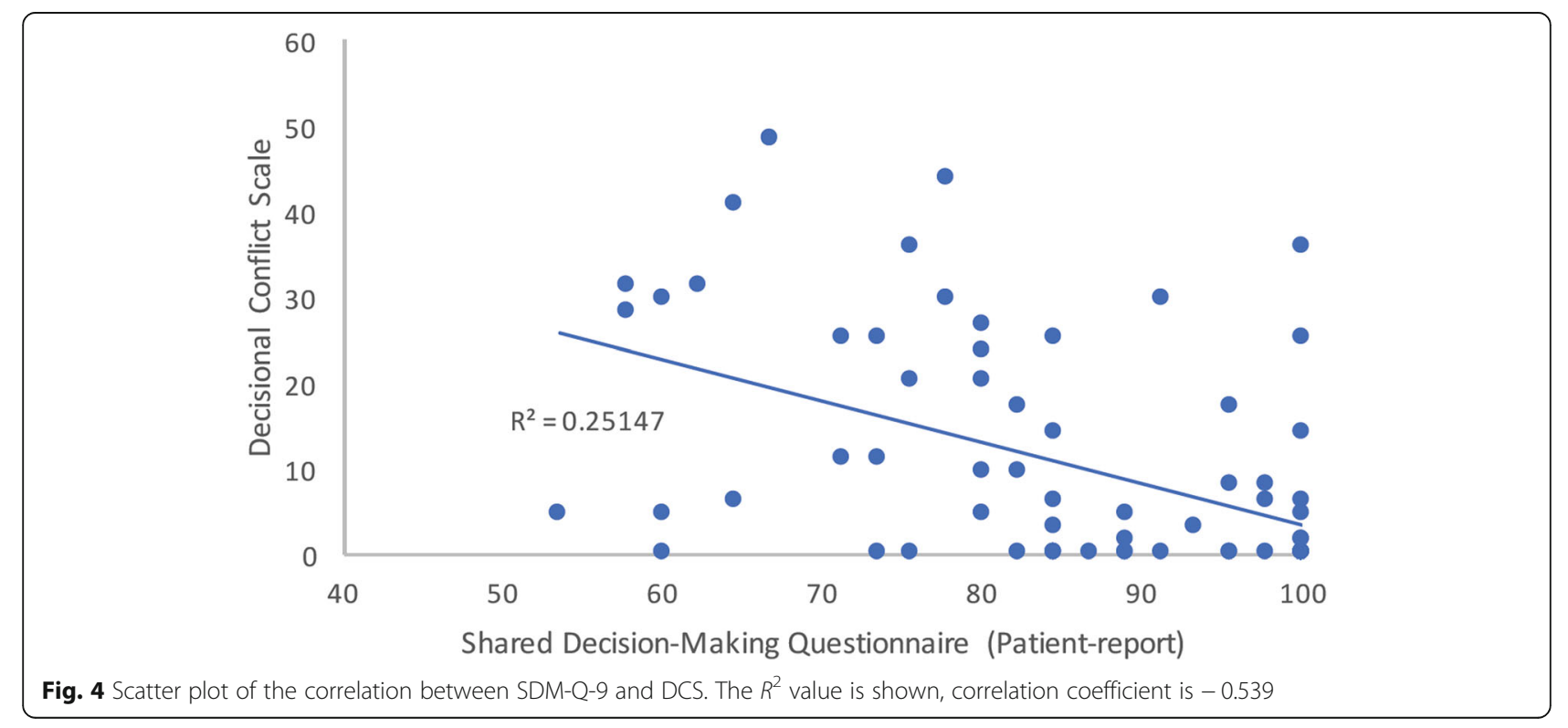




\section{Decisional Conflict by Treatment Decision}

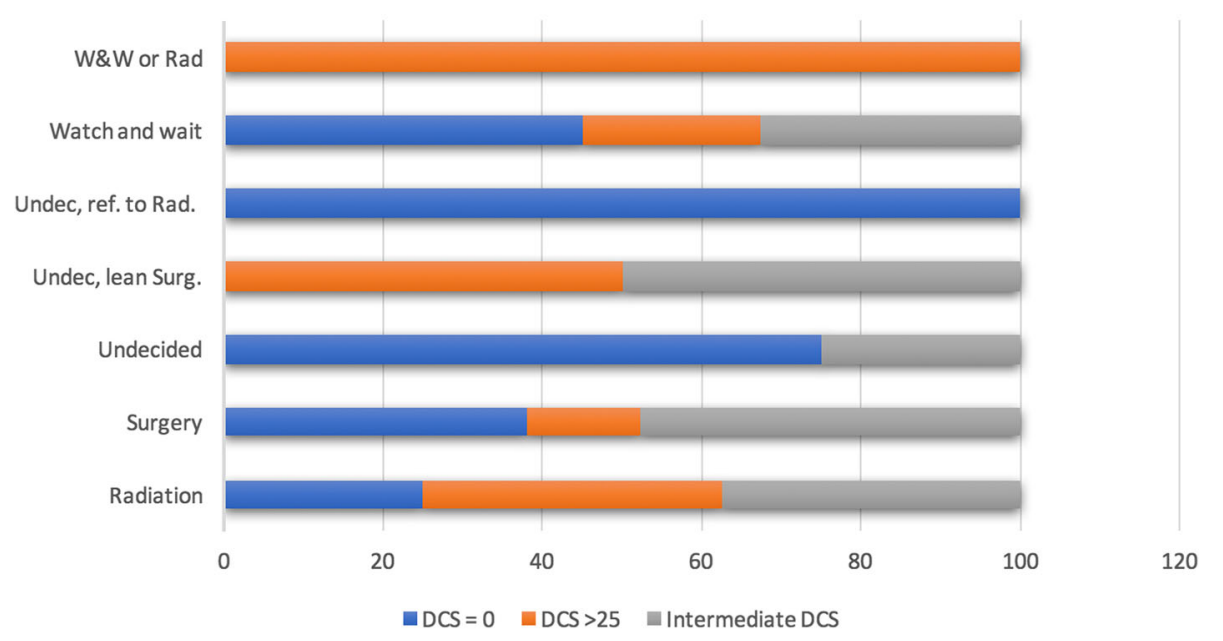

Fig. 5 Distribution of DCS score by treatment decision. Those who remained undecided about their final treatment option (i.e. W \& W or rads, undecided and leaning toward surgery) appeared to experience more decisional conflict

study may be required to elucidate the mechanism of reduced DCS.

\section{Comparison to other studies}

Data on decisional conflict and shared decision making in patients with otolaryngology disorders is limited [15, 17, 18]. These, and studies in other surgical decision-making contexts have shown that patients experience significant decisional conflict when deciding between surgical and non-surgical treatment for various conditions [16]. For instance, almost one fifth of parents considering elective pediatric surgery for their child experienced a critical level of decisional conflict, with a DCS score greater than the predefined cutoff of 25 [15]. Decisional conflict was significantly correlated with parental perception of SDM, with patients feeling more involved in the surgical decision experiencing significantly less decisional conflict. In parents specifically considering bone anchored hearing devices for their child with aural atresia, over $40 \%$ reported experiencing significant decisional conflict [18]. This has also been shown in pediatric urology, with nearly a third of parents considering hypospadias repair for their child experiencing decisional conflict [16]. The proportion of patients with significant decisional conflict in the current study compares with data on pediatric patients undergoing elective procedures [15] but is less than adult patients considering thyroidectomy for indeterminate nodules, (34\%) [17], or pediatric patients considering bone anchored hearing aids (43.5\%) [18] or otoplasty (32.8\%) [25]. The degree of conflict experienced by patients appears to vary considerably depending on the condition and ramifications of surgery.
Although research is increasingly showing that shared decision making is crucial in improving care, there are significant barriers to its implementation, both on the side of the healthcare provider and the patient. A systematic review by Legare et al. suggests that time constraints in a busy clinical practice remain the most frequent barrier to SDM cited by physicians [26]. Research does not presently exist showing that increasing SDM increases time of clinical encounters, however, so this may be a misconception. Physicians also may assume, at times based on socioeconomic status or other demographic factors, that patients may not desire involvement in the process of decision making [26]. Given we did not find that there was a difference in SDM or DCS based on these demographic factors, our study would strongly suggest providers not make such assumptions.

Patient identified barriers to shared decision making include inadequate provision of information to patients to allow them to make informed decisions [27]. Decision-aids are being developed in multiple areas to assist with this barrier, but improving information delivery in isolation is not enough. Other barriers identified include lack of patient knowledge that they may and should be involved in decisions regarding their care. This perception that the "doctor knows best", and the power imbalance perceived in the doctor-patient relationship may preclude patients from participating in decisions, thinking inclusion of their values is not needed or appropriate. In vestibular schwannoma management, the neurotologist must empower the patient to be a participant in the decision-making process, giving them "permission to participate", rather than just providing information. 


\section{Study strengths and limitations}

There are limitations to this study. The inclusion of two sites may have resulted in differing patient experiences by geographic location given differences in clinic accommodation of patients with VS. Additionally, we did not have a standardized script delivered by each provider at each visit, meaning the information presented to each patient might vary. However, this is more in keeping with what occurs in clinical practice: each patient requires directed consultation based on their presentation, and standardizing information delivery would be likely to falsely estimate the prevalence of decisional conflict in these patients. Nonetheless, the DCS scores between these two sites were not found to be significantly different in our analysis. Alternatively, the inclusion of two sites would increase the likelihood our findings could be generalizable to other skull base clinics. Most patients decided on a watch and wait approach; this may bias the degree of decisional conflict present in patients as well.

In future studies, it would be useful to follow these patients longitudinally, to determine if decisional conflict is related to the degree of decisional regret associated with decisions patients make, as seen in some previous studies [16, 22, 25].

\section{Conclusions}

Approximately one in five patients with vestibular schwannoma experience significant decisional conflict. Increasing the degree to which the patient is involved in the decisionmaking process, through shared decision-making, may decrease the difficulty patients have and improve their experience in managing this potentially debilitating condition.

\author{
Abbreviations \\ DCS: Decisional conflict scale; SDM: Shared decision making; SDM-Q- \\ 10: Patient shared decision making questionnaire; SDM-Q-Doc: Physician \\ version of the SDM-Q-10; SE: Standard error; SRS: Stereotactic radiation \\ surgery; VS: Vestibular Schwannoma
}

\section{Funding}

A portion of the funding for this study was obtained through a grant from the Nova Scotia Health Authority Research Fund, and funding a research assistant for data collection and entry.

\section{Availability of data and materials}

The datasets used and/or analyzed during the current study are available from the corresponding author on reasonable request.

\footnotetext{
Authors' contributions

MEG conceptualized the study and the patient materials and drafted the manuscript. BDW, JL, and MB supervised the study, assisted in its conception, and provided editorial feedback. PH provided expertise on SDM/DCS surveys, performed manuscript revisions and assisted with study conception and grant approval. HF performed statistical analysis. EP and MM were responsible for Halifax site survey administration, data collection and entry, and revision of patient material. ALOH administered the survey at the Halifax site and assisted with manuscript editing. RG obtained ethical approval and administered the survey with data collection/entry at the Vancouver site. DPM and SW assisted with manuscript editing and survey administration. All authors read and approved the final manuscript.
}

\section{Ethics approval and consent to participate}

Ethical approval was obtained from the Nova Scotia Health Authority, Halifax Nova Scotia, and Providence Health Care, Vancouver, British Columbia.

\section{Consent for publication}

Not applicable.

\section{Competing interests}

The authors declare that they have no competing interests.

\section{Publisher's Note}

Springer Nature remains neutral with regard to jurisdictional claims in published maps and institutional affiliations.

\section{Author details}

${ }^{1}$ Division of Otolaryngology - Head and Neck Surgery, Western University and London Health Sciences Centre, 5010, 800 Commissioners Road E, London, Ontario, Canada. ${ }^{2}$ Division of Otolaryngology - Head and Neck Surgery, University of British Columbia, Vancouver, BC, Canada. ${ }^{3}$ IWK Health Center and Division of Otolaryngology - Head and Neck Surgery, Dalhousie University, Halifax, NS, Canada. ${ }^{4}$ Division of Neurosurgery, Dalhousie University, Halifax, NS, Canada. ${ }^{5}$ Division of Otolaryngology, Head and Neck Surgery, Dalhousie University, Halifax, NS, Canada. ${ }^{6}$ University of Cambridge, Cambridge, UK

Received: 3 May 2018 Accepted: 26 August 2018

Published online: 03 September 2018

\section{References}

1. Babu R, Sharma R, Bagley JH, Hatef J, Friedman AH, Adamson C. Vestibular schwannomas in the modern era: epidemiology, treatment trends, and disparities in management. J Neurosurg. 2013;119:121-30.

2. Martin TP, Senthil L, Chavda SV, Walsh R, Irving RM. A protocol for the conservative management of vestibular schwannomas. Otol Neurotol. 2009;30:381-5.

3. Stangerup SE, Caye-Thomasen P, Tos M, Thomsen J. The natural history of vestibular schwannoma. Otol Neurotol. 2006;27:547-52.

4. Strasnick B, Glasscock ME 3rd, Haynes D, McMenomey SO, Minor LB. The natural history of untreated acoustic neuromas. Laryngoscope. 1994;104:1115-9.

5. Ansari SF, Terry C, Cohen-Gadol AA. Surgery for vestibular schwannomas: a systematic review of complications by approach. Neurosurg Focus. 2012;33:E14.

6. Vachhrajani S., Fawaz C., Mathieu D. et al. (2008) Complications of gamma knife surgery: an early report from 2 Canadian centers. J Neurosurg 109 Suppl, 2-7.

7. Carlson ML, Jacob JT, Pollock BE, et al. Long-term hearing outcomes following stereotactic radiosurgery for vestibular schwannoma: patterns of hearing loss and variables influencing audiometric decline. J Neurosurg. 2013;118:579-87.

8. Elliott A, Hebb AL, Walling S, Morris DP, Bance M. Hearing preservation in vestibular schwannoma management. Am J Otolaryngol. 2015;36:526-34.

9. Limb CJ, Long DM, Niparko JK. Acoustic neuromas after failed radiation therapy: challenges of surgical salvage. Laryngoscope. 2005;115:93-8.

10. Shamisa A., Bance M., Nag S. et al. (2013) Glioblastoma multiforme occurring in a patient treated with gamma knife surgery: case report and review of the literature. J Neurosurg 119 Suppl, 816-821.

11. Pollock BE, Link MJ, Stafford SL, Parney IF, Garces YI, Foote RL. The risk of radiation-induced tumors or malignant transformation after single-fraction intracranial radiosurgery: results based on a 25-year experience. Int J Radiat Oncol Biol Phys. 2017;97:919-23.

12. O'Connor A. (1993) User Manual - Decisional Conflict Scale.

13. Owings MF, Kozak LJ. Ambulatory and inpatient procedures in the United States, 1996. Vital Health Stat 13. 1998;139:1-119.

14. Joosten EA, DeFuentes-Merillas $L$, de Weert GH, Sensky T, van der Staak CP, de Jong CA. Systematic review of the effects of shared decision-making on patient satisfaction, treatment adherence and health status. Psychother Psychosom. 2008;77:219-26.

15. Chorney J, Haworth R, Graham ME, Ritchie K, Curran JA, Hong P. Understanding shared decision making in pediatric otolaryngology. Otolaryngol Head Neck Surg. 2015;152:941-7.

16. Lorenzo AJ, Braga LH, Zlateska B, et al. Analysis of decisional conflict among parents who consent to hypospadias repair: single institution prospective study of 100 couples. J Urol. 2012;188:571-5. 
17. Taylor BA, Hart RD, Rigby MH, Trites J, Taylor SM, Hong P. Decisional conflict in patients considering diagnostic thyroidectomy with indeterminate fine needle aspirate cytopathology. J Otolaryngol Head Neck Surg. 2016;45:16.

18. Graham ME, Haworth R, Chorney J, Bance M, Hong P. Decisional conflict in parents considering bone-anchored hearing devices in children with unilateral aural atresia. Ann Otol Rhinol Laryngol. 2015;124:925-30.

19. Smith MY, Winkel G, Egert J, Diaz-Wionczek M, DuHamel KN. Patientphysician communication in the context of persistent pain: validation of a modified version of the patients' perceived involvement in care scale. J Pain Symptom Manag. 2006;32:71-81.

20. Scholl I, Kriston L, Dirmaier J, Buchholz A, Harter M. Development and psychometric properties of the shared decision making questionnaire-physician version (SDM-Q-doc). Patient Educ Couns. 2012;88:284-90.

21. Carr MM, Derr JB, Karikari K. Decisional conflict and regret in parents whose children undergo tonsillectomy. Otolaryngol Head Neck Surg. 2016;155:863-8.

22. Ritchie KC, Chorney J, Hong P. Parents' decisional conflict, selfdetermination and emotional experiences in pediatric otolaryngology: a prospective descriptive-comparative study. Int I Pediatr Otorhinolaryngol. 2016;86:114-7.

23. Jacob A, Robinson LL Jr, Bortman JS, Yu L, Dodson EE, Welling DB. Nerve of origin, tumor size, hearing preservation, and facial nerve outcomes in 359 vestibular schwannoma resections at a tertiary care academic center. Laryngoscope. 2007;117:2087-92.

24. Hong P, Maguire E, Gorodzinsky AY, Curran JA, Ritchie K, Chorney J. Shared decision-making in pediatric otolaryngology: parent, physician and observational perspectives. Int J Pediatr Otorhinolaryngol. 2016;87:39-43.

25. Hong P, Gorodzinsky AY, Taylor BA, Chorney JM. Parental decision making in pediatric otoplasty: the role of shared decision making in parental decisional conflict and decisional regret. Laryngoscope. 2016;126(Suppl 5):S5-S13.

26. Legare F, Ratte S, Gravel K, Graham ID. Barriers and facilitators to implementing shared decision-making in clinical practice: update of a systematic review of health professionals' perceptions. Patient Educ Couns. 2008:73:526-35.

27. Joseph-Williams N, Elwyn G, Edwards A. Knowledge is not power for patients: a systematic review and thematic synthesis of patient-reported barriers and facilitators to shared decision making. Patient Educ Couns. 2014:94:291-309

Ready to submit your research? Choose BMC and benefit from:

- fast, convenient online submission

- thorough peer review by experienced researchers in your field

- rapid publication on acceptance

- support for research data, including large and complex data types

- gold Open Access which fosters wider collaboration and increased citations

- maximum visibility for your research: over $100 \mathrm{M}$ website views per year

At $\mathrm{BMC}$, research is always in progress.

Learn more biomedcentral.com/submissions 